\title{
Oral lichenoid lesions: distinguishing the benign from the deadly
}

\author{
Susan Müller \\ Professor Emeritus, Emory University School of Medicine: Atlanta Oral Pathology, Decatur, GA, USA
}

\begin{abstract}
Oral lichen planus is a chronic inflammatory disease of unknown etiology or pathogenesis with varied disease severity that waxes and wanes over a long period of time. Although a common oral mucosal disease, accurate diagnosis is often challenging due to the overlapping clinical and histopathological features of oral lichen planus and other mucosal diseases. Other immune-mediated mucocutaneous diseases can exhibit lichenoid features including mucous membrane pemphigoid, chronic graft-versus-host disease, and discoid lupus erythematosus. Reactive changes to dental materials or to systemic medications can mimic oral lichen planus both clinically and histologically. In these situations the clinical presentation can be useful, as oral lichen planus presents as a multifocal process and is usually symmetrical and bilateral. Dysplasia of the oral cavity can exhibit a lichenoid histology, which may mask the potentially premalignant features. Proliferative verrucous leukoplakia, an unusual clinical disease, can often mimic oral lichen planus clinically, requiring careful correlation of the clinical and pathologic features.

Modern Pathology (2017) 30, S54-S67; doi:10.1038/modpathol.2016.121
\end{abstract}

Diagnosing oral lichen planus can be challenging due to various conditions that have overlapping features-both clinically and histologically. The terminology, classification, and diagnosis of oral lichenoid lesions have been explored, debated, and analyzed for decades. ${ }^{1-4}$ Many names have been used in the literature, which contribute to the confusion surrounding terminology, impeding our ability to develop effective approaches to diagnosis and management. The differential diagnosis of oral lichenoid lesions includes other immune-mediated mucocutaneous diseases, and reactive and inflammatory conditions.

The malignant potential of oral lichen planus is fraught with controversy due to lack of reliable histologic diagnostic criteria and in some reports the diagnosis of oral lichen planus was made exclusively on the clinical presentation without histologic confirmation. ${ }^{5-11}$ Oral dysplasia can exhibit lichenoid features masking the potentially cancerous component. ${ }^{12,13}$ The presence of dysplasia in an oral lichenoid lesion precludes a diagnosis of oral lichen planus and rendering the diagnosis of oral lichen planus with dysplasia can result in patient mismanagement. Proliferative verrucous leukoplakia is an uncommon oral disease that, due to the multi-

Correspondence: Dr S Müller, DMD, MS, Atlanta Oral Pathology, 2701 North Decatur Road, Decatur, GA 30033, USA.

E-mail: smullerdmd@gmail.com

Received 30 May 2016; revised 31 May 2016; accepted 2 June 2016 focal presentation, can mimic oral lichen planus clinically and histologically, particularly in its early stages. ${ }^{1,2,14,15}$ However, there are distinct architectural changes in proliferative verrucous leukoplakia including hyperkeratotic verrucous epithelium that can aid a pathologist.

An accurate diagnosis of oral lichen planus and oral lichen planus mimics cannot be made in a vacuum and it is essential that clinicians provide patient information including site, symptoms, and other relevant information with the biopsy requisition. Correlation of the clinical presentation coupled with the histologic features is essential when examining oral biopsies with lichenoid features.

\section{Oral lichen planus and benign lichenoid lesions}

Oral Lichen Planus

Oral lichen planus is a relatively common mucocutaneous disease with an estimated prevalence of 0.22 to $5 \%$ worldwide. ${ }^{16}$ Oral lichen planus is more prevalent in females and most often presents in the fourth to eighth decade ${ }^{16-19}$ Although rare, pediatric involvement occurs and in one study $18 \%$ of the childhood lichen planus cases had oral involvement. ${ }^{20,21}$ Although up to $60 \%$ of patients with cutaneous lichen planus have oral manifestation, only a minority of oral lichen planus 

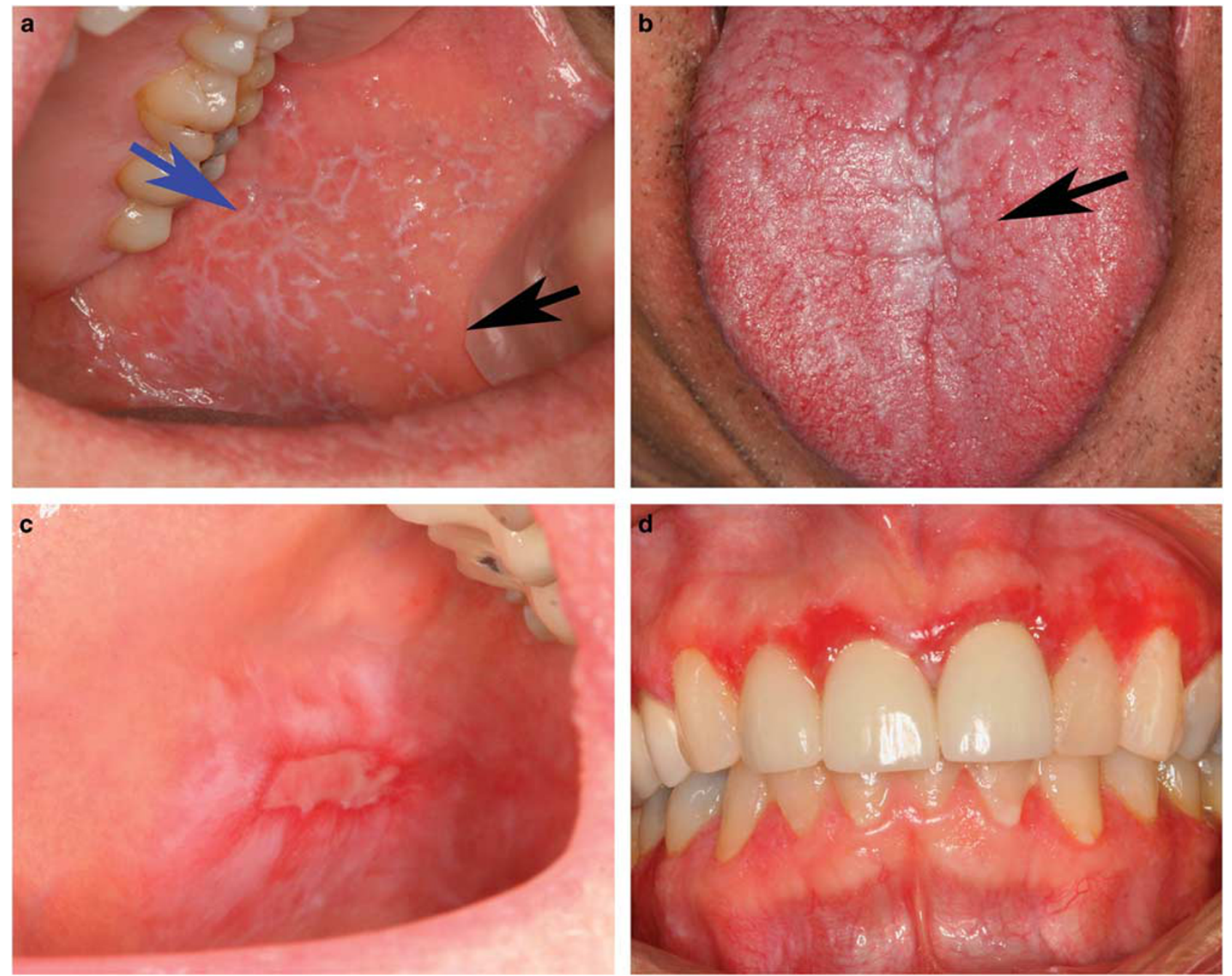

Figure 1 Clinical patterns of oral lichen planus. Reticular (blue arrow) and papular (black arrow) (a); plaque pattern on dorsal tongue (arrow) (b); erosive (c); atrophic form presenting as desquamative gingivitis (d).

patients (15\%) develop cutaneous lichen planus. ${ }^{18,22}$ Other anatomic sites of involvement include the genitalia and both peno-gingival syndrome and vulvo-vaginal-gingival syndrome have been reported in up to $20 \%$ of patients with oral lichen planus. ${ }^{18}$ Esophageal involvement can occur with oral lichen planus but is unusual. Lichen planus can involve the scalp (resulting in scarring alopecia), nails, and conjunctivae. ${ }^{23}$

Oral lichen planus is a multifocal disease and presents more or less in a symmetrical distribution, typically affecting the buccal mucosa, tongue, lips, gingiva, and rarely the palate and floor of mouth. ${ }^{1,16,24}$ In the oral cavity, there are six clinical presentations of oral lichen planus: reticular, atrophic (erythematous), erosive, papular, plaque, and bullous (Figure 1a-d). Many of these patterns occur simultaneously or sequentially. The most characteristic clinical presentation is intersecting white lines (striae) with or without erythema that can be pigmented in some ethnic groups. ${ }^{16-18,25}$
Plaque-like oral lichen planus is most commonly found on the dorsal tongue and has been reported to be more common in cigarette smokers. ${ }^{25}$ Erosive oral lichen planus when associated with severe ulcerations can mask the typical oral lichen planus striae but careful clinical examination will often show the recognizable features of oral lichen planus. Gingival involvement by oral lichen planus usually presents as desquamative gingivitis and is clinically indistinguishable from other diseases including mucous membrane pemphigoid and pemphigus vulgaris. ${ }^{1,2}$ The facial gingiva is most commonly affected but in severe cases both palatal and lingual gingival mucosa can be involved.

Rendering the correct diagnosis requires good communication with the clinician who hopefully is familiar with both the clinical and histologic features of lichen planus. As the diagnosis of lichen planus requires evaluation of the basement membrane zone (BMZ), biopsies of oral lichen planus must include intact, full thickness epithelium. A biopsy of only the 

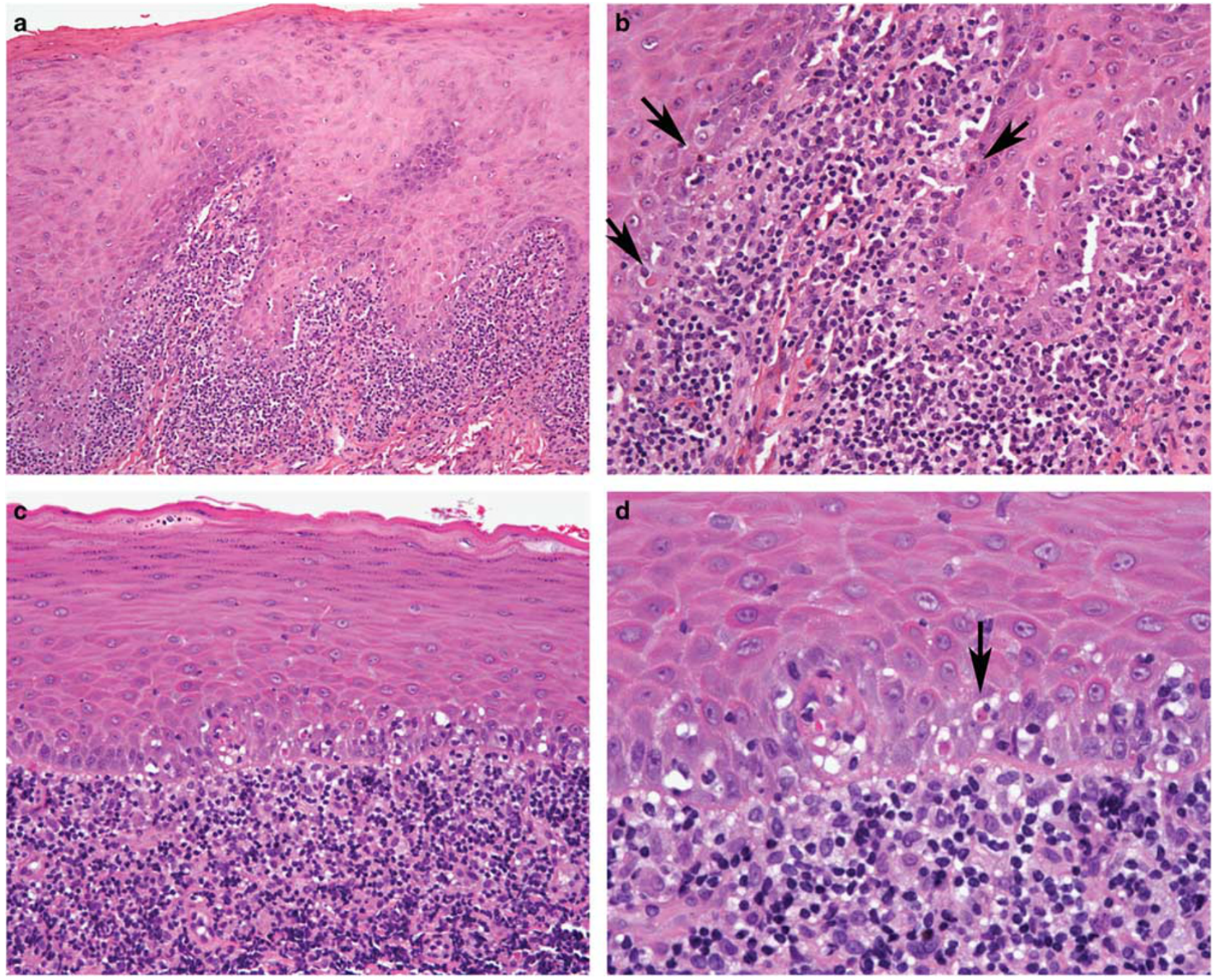

Figure 2 Histopathologic features of the reticular form of oral lichen planus. Oral mucosal stratified squamous epithelium exhibits a thickened surface layer of parakeratin, mild acanthosis and 'saw-tooth' rete ridge morphology, and a dense band-like chronic inflammatory cell infiltrate in the superficial lamina propria (a, H\&E stain, original magnification $\times 100$ ). Hydropic degeneration in basal cells are apparent with dissolution of the basement membrane. Lymphocyte-mediated injury of oral mucosal stratified squamous epithelium, with keratinocyte apoptosis represented as a colloid (Civatte) body (arrows) (b, H\&E stain, original magnification $\times 250$ ). Oral lichen planus from an atrophic area with flattened rete. A thin eosinophilic band adjacent to the basal cell layer is present as well as the band-like lymphocytic infiltrate (c, H\&E stain, original magnification $\times 250)$. On higher power, both dyskeratosis (arrow) and hydropic basal cell degeneration is present (d, H\&E stain, original magnification $\times 400)$.

ulcerative component will not show the interface changes necessary for diagnosis.

The American Academy of Oral and Maxillofacial Pathology recently published a position paper on the histologic diagnostic guidelines of oral lichen planus modifying the criteria reported by van der Meij and van der Waal. ${ }^{26,27}$ The microscopic features of oral lichen planus are highly variable, as biopsies from hypertrophic, atrophic, or erosive sites can exhibit different histologic features. Interface mucositis is a hallmark of oral lichen planus. Hydropic degeneration of the basal cells with scattered dyskeratotic keratinocytes (Civatte, colloid, hyaline, or cytoid bodies) along the epithelial interface is seen (Figure 2a-d). ${ }^{1,27}$ At the basement zone, hugging the basal cells is a band-like, predominately T-lymphocyte infiltrate. A 'sawtooth' pattern of the rete can be observed in oral lichen planus but this histologic finding is more common in cutaneous LP. ${ }^{16}$ The epithelium can also appear acanthotic or atrophic corresponding to the clinical presentation. In general, the inflammation is superficial rather than deep and perivascular inflammation is not typically present. Other histologic findings include a homogeneous eosinophilic deposit at the epitheliallamina propria interface, melanosis, and melanin incontinence with associated melanophages (Figure 3a and b). The presence of melanin is not specific to oral lichen planus and can be seen in oral biopsies from other oral inflammatory disorders. ${ }^{28}$ Biopsies of erosive oral lichen planus lack many of the histologic hallmarks of lichen planus and 

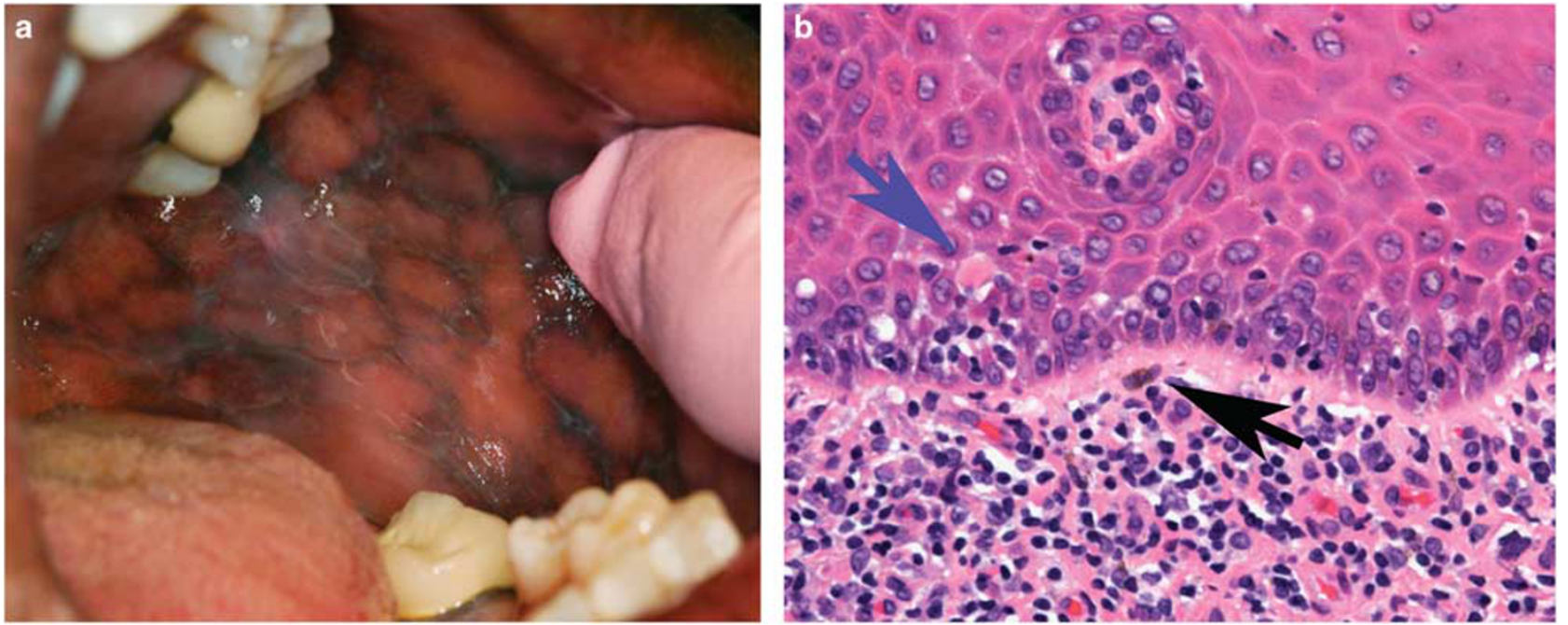

Figure 3 Pigmented oral lichen planus. In dark-skinned individuals, pigmentation can be seen associated with the reticular pattern. (a) Melanosis and melanin incontinence with associated melanophages can sometimes be found, especially in biopsies from individuals with dark complexions (black arrow) along with the usual microscopic findings of oral lichen planus including Civatte bodies (blue arrow) (b, H\&E stain, original magnification $\times 400$ ).
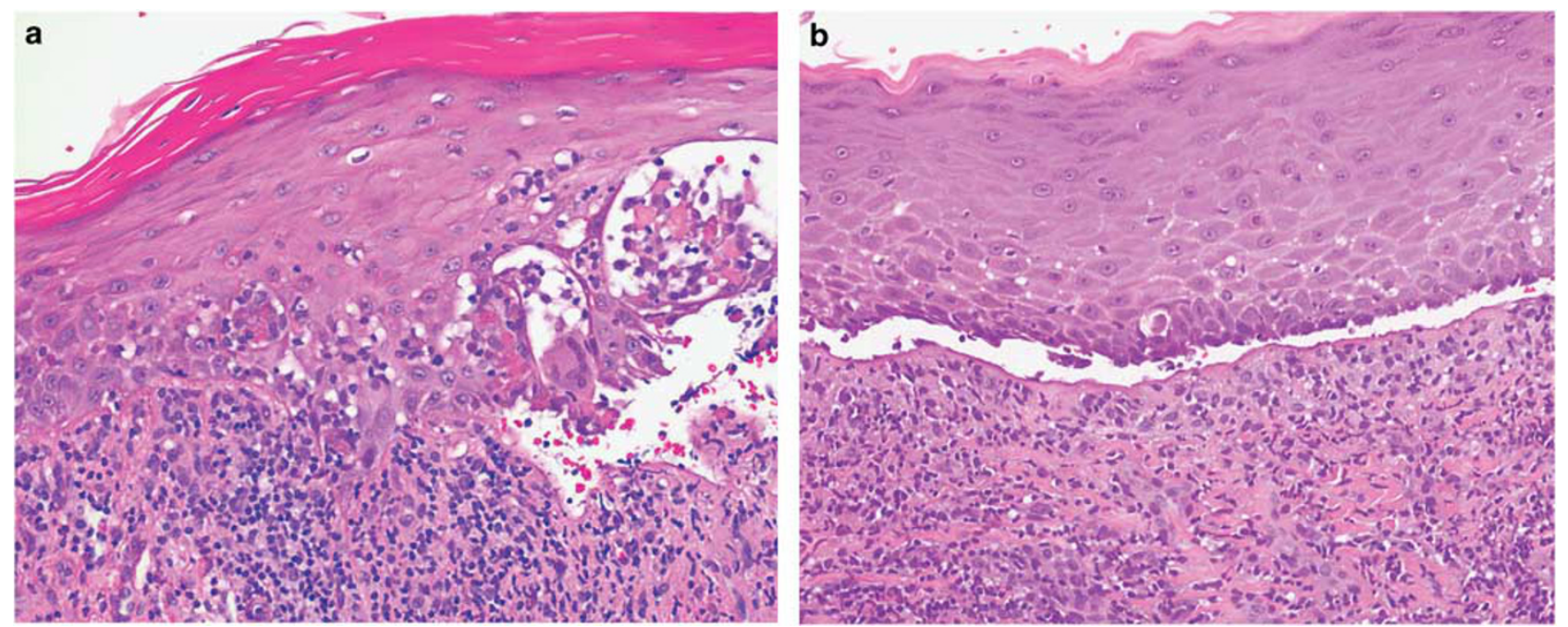

Figure 4 Erosive oral lichen planus. This biopsy from the buccal mucosa exhibits a sub-basal separation with numerous colloid bodies. Often in the area of the ulceration a mixed inflammation is seen and the more diagnostic areas of oral lichen planus are adjacent to the ulcer. (a, H\&E stain, original magnification $\times 250$ ); biopsies of gingival oral lichen planus can mimic mucous membrane pemphigoid, although demonstration of hydropic basal cell degeneration and colloid bodies can often be seen after careful evaluation. Direct immunofluorescence is sometimes needed to distinguish these two entities. (b, H\&E stain, original magnification $\times 250$ ).

arriving at a definitive diagnosis can be difficult (Figure 4a and b).

Direct immunofluorescence of oral lichen planus is nonspecific and includes shaggy fibrin and/or complement (C3) deposits in a granular or linear pattern along the BMZ. ${ }^{29,30}$ IgM-positive colloid bodies can also be identified. This direct immunofluorescence pattern can be seen in other inflammatory conditions, as well as in premalignant and malignant oral lesions. ${ }^{31}$ Therefore, direct immunofluorescence is not necessary to make the diagnosis of oral lichen planus, although direct immunofluorescence can be useful to distinguish oral lichen planus from other vesiculobullous diseases such as mucous membrane pemphigoid and chronic ulcerative stomatitis (Table 1). ${ }^{29,30}$ Indirect immunofluorescence is negative in oral lichen planus.

\section{Benign Lichenoid Lesions}

Oral lichenoid lesions are well documented and can have a variety of etiologies. Table 1 lists the various histologic mimics of oral lichen planus and 
Table 1 Distinguishing histologic mimics of oral lichen planus

\begin{tabular}{|c|c|c|c|}
\hline Disease & Clinical & Histology & Immunopathology \\
\hline Oral lichen planus & $\begin{array}{l}\text { Multifocal oral involvement with } \\
\text { roughly symmetrical distribution. Red } \\
\text { and white lesions presenting as } \\
\text { reticular, atrophic, erosive, or plaque } \\
\text { and rarely bullous. }\end{array}$ & $\begin{array}{l}\text { Usually hyperparakeratosis but may } \\
\text { have orthokeratosis. Epithelium may } \\
\text { be atrophic, acanthotic and have 'saw- } \\
\text { tooth' rete. Basal cell degeneration } \\
\text { with leukocytic exocytosis and } \\
\text { presence of Civatte bodies. Band-like } \\
\text { or patchy predominately lymphocytic } \\
\text { infiltrate subjacent to the basal cells. } \\
\text { Subepithelial clefting can be present in } \\
\text { erosive OLP. Gingival biopsies may } \\
\text { contain plasma cells in addition to } \\
\text { lymphocytes. No epithelial dysplasia } \\
\text { or verrucous architectural change. }\end{array}$ & $\begin{array}{l}\text { DIF: usually negative but may see } \\
\text { shaggy deposits of fibrin and/or } \\
\text { complement (C3) at BMZ and IgM- } \\
\text { positive colloid bodies. } \\
\text { IIF: negative }\end{array}$ \\
\hline
\end{tabular}

Mucous membrane Clinically can mimic OLP especially pemphigoid when presenting as desquamative gingivitis. Positive Nikolsky sign.

Oral lichenoid drug reaction

ULCR_amalgam $\quad \begin{aligned} & \text { Unilateral lesion in direct contact with } \\ & \text { dental amalgam. }\end{aligned}$

OLCR—cinnamon

Lupus erythematosus

Chronic graft vs host disease

Chronic ulcerative stomatitis

Oral dysplasia
May present as a single lesion. Temporal relationship with medications (see Table 2) although OLDR onset ranges from weeks to years.

dental amalgam.

White plaques or erythema occurring in the area of contact with resolution after discontinuing product.

Less symmetry with central ulceration surrounded by radiating striae.
Presents $>6$ months post allogeneic bone marrow transplant. Mimics OLP clinically and can be seen throughout oral cavity

Oral findings indistinguishable from OLP and MMP
Subepithelial clefting with

detachment from the lamina propria.

No hydropic degeneration of the basal cells or colloid bodies. Inflammation often patchy, variable and contains lymphocytes, plasma cells and possibly eosinophils.

Similar to OLP but may have a higher number of apoptotic keratinocytes. The inflammation may be more diffuse rather than band-like and contain plasma cells and eosinophils. Perivascular chronic inflammation often seen.

Histology overlaps with OLP; however, may see tertiary lymphoid follicles.

Epithelial acanthosis with elongated rete ridges, interface mucositis, and diffuse mixed inflammation with deep perivascular infiltrates.

May see atrophy of the epithelial rete with colloid bodies and thickened basement membrane. Lamina propria is edematous and inflammation varies from sparse to lymphocyte rich.

Melanin incontinence may be present. Both superficial and deep inflammatory (perivascular) infiltrates.

Similar to OLP including basal cell degeneration and colloid bodies. At times the chronic inflammatory infiltrate sparser and mixed.

Similar to OLP. Biopsies from ulcerative sites have a mixed inflammatory infiltrate

Band-like mostly lymphocytic infiltrate can be seen in some oral dysplasia mimicking OLP on lowpower microscopy. May see other features of OLP focally in some cases including interface mucositis and colloid bodies. Closer examination demonstrates the features of dysplasia including cytologic atypia
DIF: continuous linear deposits of IgG, IgM, or IgA, and complement (C3) along the BMZ

IIF: often negative but using salt-split skin can increase sensitivity

DIF: shaggy deposits of fibrin at BMZ and IgM positive colloid bodies similar to OLP.

IIF: rarely may detect circulating antibodies directed to the basal cells with an annular fluorescent distribution termed 'string of pearls' pattern.

DIF: similar to OLP

IIF: negative

DIF: similar to OLP

IIF: negative

DIF: Granular or shaggy deposits of IgG, IgM, or C3 at BMZ

IIF: negative in discoid LE; systemic LE commonly ANA, anti-doublestranded DNA, anti-SM, RNP, Ro/SSA and La/SSB, anti-antiphospholipid, and cardiolipin positive

DIF: similar to OLP

IIF: negative

DIF: IgG in the nuclei of basal and parabasal epithelial cells in a speckled and/or granular pattern (SES-ANA pattern).

IIF: SES-ANA positive on monkey or guinea pig esophagus

DIF: cannot be used to distinguish oral dysplasia from OLP single lesion, except for proliferative verrucous leukoplakia

Abbreviations: BMZ, basement membrane zone; DIF, direct immunofluorescence; IIF, indirect immunofluorescence; LE, lupus erythematosus; MMP, mucous membrane pemphigoid; OLCR, oral lichenoid contact reaction; OLDR, oral lichenoid drug reaction; OLP, oral lichen planus; SES-ANA, stratified epithelium specific-antinuclear antibody. ${ }^{1-4,26}$ 

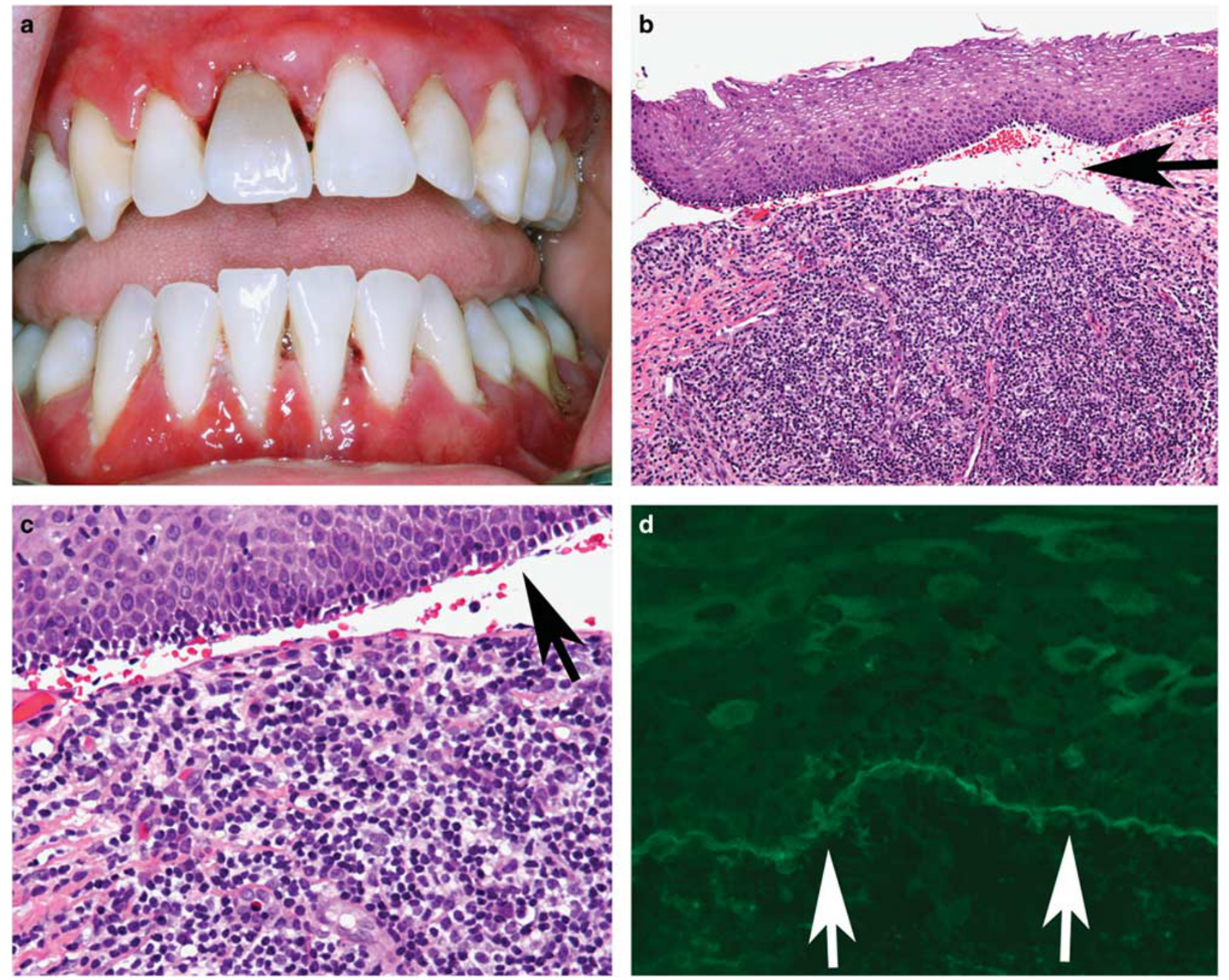

Figure 5 Mucous membrane pemphigoid presenting as desquamative gingivitis similar to the atrophic form of oral lichen planus. This is one of the most common clinical presentations of oral mucous membrane pemphigoid but can also be seen in pemphigus vulgaris and oral lichen planus (a). Histopathological features of mucous membrane pemphigoid, showing characteristic sub-epithelial clefting (arrow) (b, H\&E stain, original magnification $\times 100$ ). Unlike oral lichen planus, the basal cells are intact (arrow) and the superficial lamina propria contains a sparse to moderate inflammatory cell infiltrate consisting of lymphocytes and plasma cells. (c, H\&E stain, original magnification $\times 400$ ). Direct immunofluorescence of perilesional tissue from a patient with mucous membrane pemphigoid demonstrates a continuous linear band of IgG at the basement membrane zone (BMZ, arrows) (d).

compares the clinical features, histology, and immunopathology with oral lichen planus. Mucous membrane pemphigoid is a heterogeneous group of subepithelial blistering autoimmune diseases that mostly affect the mucosa and can result in scarring. Mucous membrane pemphigoid and oral lichen planus can be clinical mimics, in particular when presenting as desquamative gingivitis, but direct immunofluorescence can be helpful in separating between these two diseases (Figure 5a-d). ${ }^{1,2}$ Most patients with mucous membrane pemphigoid will have continuous linear deposits of IgG, IgM, or IgA and complement (C3) along the BMZ on direct immunofluorescence. ${ }^{32}$ Indirect immunofluorescence is less sensitive in mucous membrane pemphigoid as patients exhibit inconsistently circulating autoantibodies directed against the BMZ, although the use of a salt-split skin substrate can increase IIF sensitivity. ${ }^{33,34}$ Immunoblot assays and enzymelinked immunosorbent assay increases the sensitivity and specificity of detected autoantibodies in mucous membrane pemphigoid sera. ${ }^{35}$

Systemic drug exposure can cause oral lichenoid drug reactions. ${ }^{1,3,4} \mathrm{~A}$ variety of medications have been implicated in oral lichenoid drug reaction (Table 2); the most frequently reported are nonsteroidal anti-inflammatory drugs, anti-hypertensives, and anti-malarials. The pathogenesis and exact incidence of oral lichenoid drug reaction is unknown. One study theorizes that prone patients have polymorphisms of the cytochrome P450 enzymes resulting in altered metabolism of some medications but this proposal requires confirmational studies. ${ }^{36}$ The oral lichenoid drug reaction is 
Table 2 Causative agents in oral lichenoid reactions

\begin{tabular}{|c|c|}
\hline $\begin{array}{l}\text { Antianxiety/psychotropic } \\
\text { agents }\end{array}$ & $\begin{array}{l}\text { Nonsteroidal anti-inflammatory } \\
\text { drugs }\end{array}$ \\
\hline Benzodiazepine & Aspirin \\
\hline Lithium & Diclofenac \\
\hline Tricyclic antidepressants & Ibuprofen \\
\hline Antibiotics & Indomethacin \\
\hline Isoniazid & Naproxen \\
\hline Rifampin & Miscellaneous \\
\hline Streptomycin & Allopurinol \\
\hline Tetracycline & Bismuth \\
\hline Anticonvulsants & Dapsone \\
\hline Carbamazepine & Gold salts \\
\hline Phenytoin & Penicillamine \\
\hline Valproate & Sulfasalazine \\
\hline Antidiabetics & Statins \\
\hline Insulin & Fluvastatin \\
\hline Sulfonylureas & Lovastatin \\
\hline Glipizide, Glyburide & Pravastatin \\
\hline Tolbutamide & Simvastatin \\
\hline Antifungals & Dental metals \\
\hline Amphotericin B & $0.1 \%$ Mercury chloride \\
\hline Ketoconazole & $1 \%$ Ammoniated mercury \\
\hline Antihypertensives & Beryllium \\
\hline Atenolol & Bismuth \\
\hline Captopril & Chromium \\
\hline Chlorothiazide & Cobalt \\
\hline Enalapril & Copper \\
\hline Furosemide & Gold \\
\hline Hydroclorothiazide & Metallic mercury \\
\hline Metoprolol & Nickel \\
\hline Propranolol & Palladium \\
\hline Antimalarials & Silver \\
\hline Chloroquine & Tin \\
\hline Hydroxychloroquine & Other dental materials \\
\hline Quinacrine & Acrylate compounds \\
\hline Quinidine & Composite \\
\hline Antiretrovirals & Glass ionomer \\
\hline Zidovudine & Porcelain \\
\hline Biologic agents & Flavoring agents \\
\hline Obinutuzumab & Balsam of Peru \\
\hline Tumor necrosis factor $\alpha$ & Cinnamon (cinnamic \\
\hline $\begin{array}{l}\text { (TNF- } \alpha \text { ) inhibitors } \\
\text { Infliximab }\end{array}$ & $\begin{array}{l}\text { aldehyde) } \\
\text { Eugenol }\end{array}$ \\
\hline Certolizumab & Menthol \\
\hline Etanercept & Mint (mentha piperita) \\
\hline Abatacept & Tartar control toothpaste \\
\hline
\end{tabular}

References ${ }^{1,2,4,26,36}$

more common in adults and is rarely reported in children. An oral lichenoid drug reaction generally presents as a single lesion unlike oral lichen planus. Establishing a relationship to the offending medication can be difficult, as the time interval between initiation of medication and the development of oral lichenoid drug reaction can range from weeks to a year or more. Table 1 highlights the histologic differences between oral lichenoid drug reaction and oral lichen planus including a more diffuse mixed inflammatory infiltrate with perivascular inflammation (Figure 6a). The microscopic findings in oral lichenoid drug reactions are considered nonspecific and clinical information including a temporal relationship with the use of systemic medications and resolution of the lesions following drug discontinuation aid in the diagnosis of an oral lichenoid drug reaction.

Oral lichenoid contact reactions have been described and are associated with a variety of topical agents including dental materials and flavoring agents (Table 2). ${ }^{1,3,4,37}$ Lichenoid lesions can occur from mucosa in direct contact to amalgam restorations and are seen most commonly on the lateral tongue or buccal mucosa. ${ }^{37,38}$ The histology often shows tertiary lymphoid follicle formation composed of B cells containing follicular dendritic cells surrounded by $\mathrm{T}$ cells and macrophages similar to tonsils (Figure 6b). ${ }^{1,2,38}$ Unlike oral lichen planus, oral lichenoid contact reaction to amalgam are usually single and will resolve with removal of the amalgam. Oral lichenoid contact reaction to cinnamoncontaining products such as gums and candies can cause a hypersensitivity reaction termed cinnamon stomatitis. ${ }^{1-3}$ The histology overlaps with oral lichen planus, although in cinnamon stomatitis marked epithelial acanthosis with elongated rete ridges and a mixed inflammatory cell infiltrate with perivascular inflammation is present (Figure 7a and b). ${ }^{39,40}$ Similar to oral lichenoid contact reaction to amalgam, discontinuing the cinnamon product will quickly result in resolution of the mucosal lesions.

Both discoid lupus erythematosus and systemic lupus erythematosus can have oral manifestations that are similar in appearance to oral lichen planus., ${ }^{1,3,4}$ The oral mucosa can be affected in up to $25 \%$ of patients with lupus erythematosus. Intraoral lupus erythematosus lesions are not distributed in a symmetrical pattern such as oral lichen planus and are found throughout the oral cavity including hard palate, buccal mucosa, lip, and gingiva. ${ }^{4,41}$ The lesions typically have a central atrophic or ulcerated area surrounded by radiating white striae. The margins of the lesion are less defined than oral lichen planus. Most patients with oral manifestations of lupus erythematosus will also have concurrent cutaneous lesions and other characteristics of lupus erythematosus such as photosensitivity. ${ }^{41}$

The histologic features of oral lupus erythematosus is not specific and overlaps with other oral lichenoid lesions including oral lichen planus, oral lichenoid contact reaction, and oral lichenoid drug reaction. ${ }^{42,43}$ The epithelium can range from atrophic to hyperplastic with keratin plugging and a thickened basement membrane. The inflammation in the lamina propria can be mixed or lymphocyte rich and range from paucicellular to band like, similar to oral lichen planus. Perivascular inflammatory infiltrates are usually present but this finding overlaps with oral lichenoid contact reaction and oral lichenoid drug reaction. Civatte bodies and interface mucositis can be observed and melanin incontinence adjacent to the epithelium may be seen. Direct immunofluorescence of perilesional tissue of oral systemic lupus erythematosus and discoid lupus erythematosus shows granular or shaggy deposits of IgG, IgM, and/or C3 in the BMZ. ${ }^{3,4,42}$ These findings 

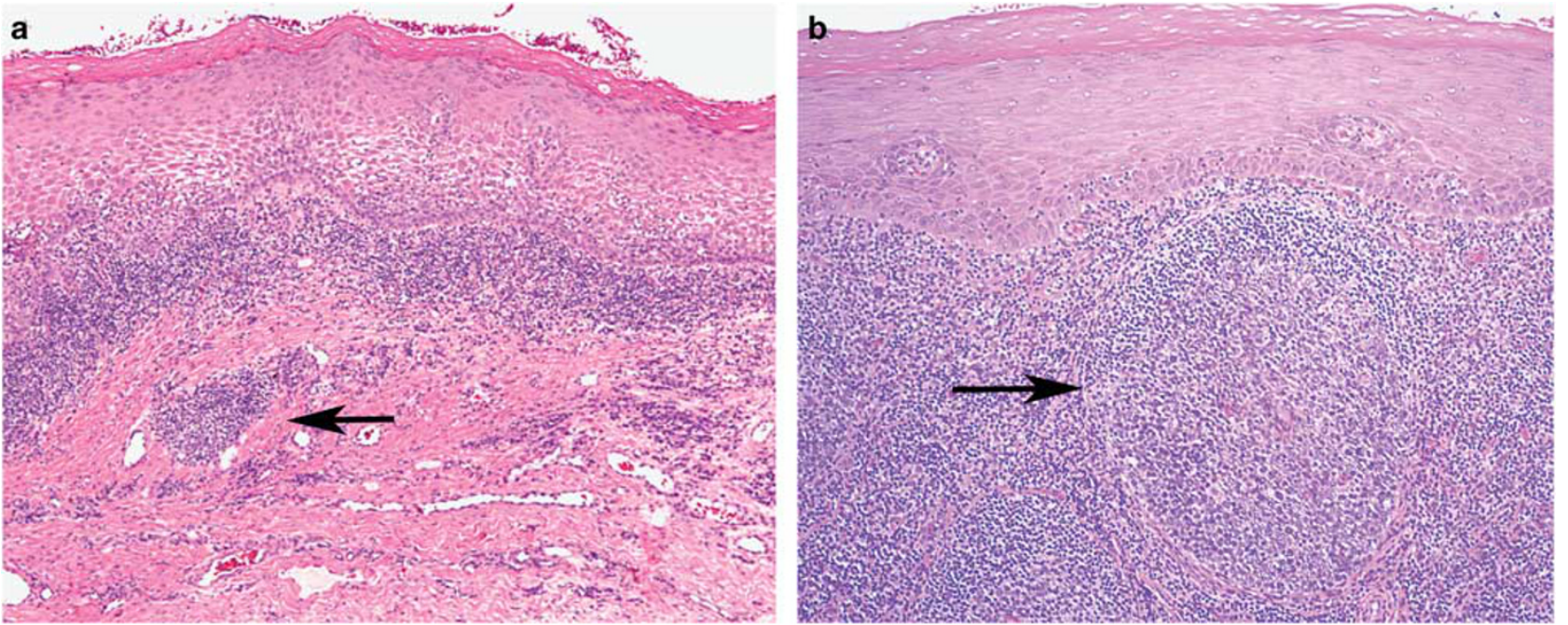

Figure 6 Oral lichenoid drug reaction. Acanthosis and inflammatory exocytosis is seen along with perivascular inflammation (arrow). The inflammation in oral lichenoid drug reactions generally extends deeper into the lamina propria than oral lichen planus. However, these microscopic findings are relatively non-specific $(\mathbf{a}, \mathrm{H} \& \mathrm{E}$ stain, original magnification $\times 100)$. Oral lichenoid contact reaction to dental amalgam often has a dense lymphocytic infiltrate, which can form tertiary lymphoid follicles (arrow) (b, H\&E stain, original magnification $\times 100)$.
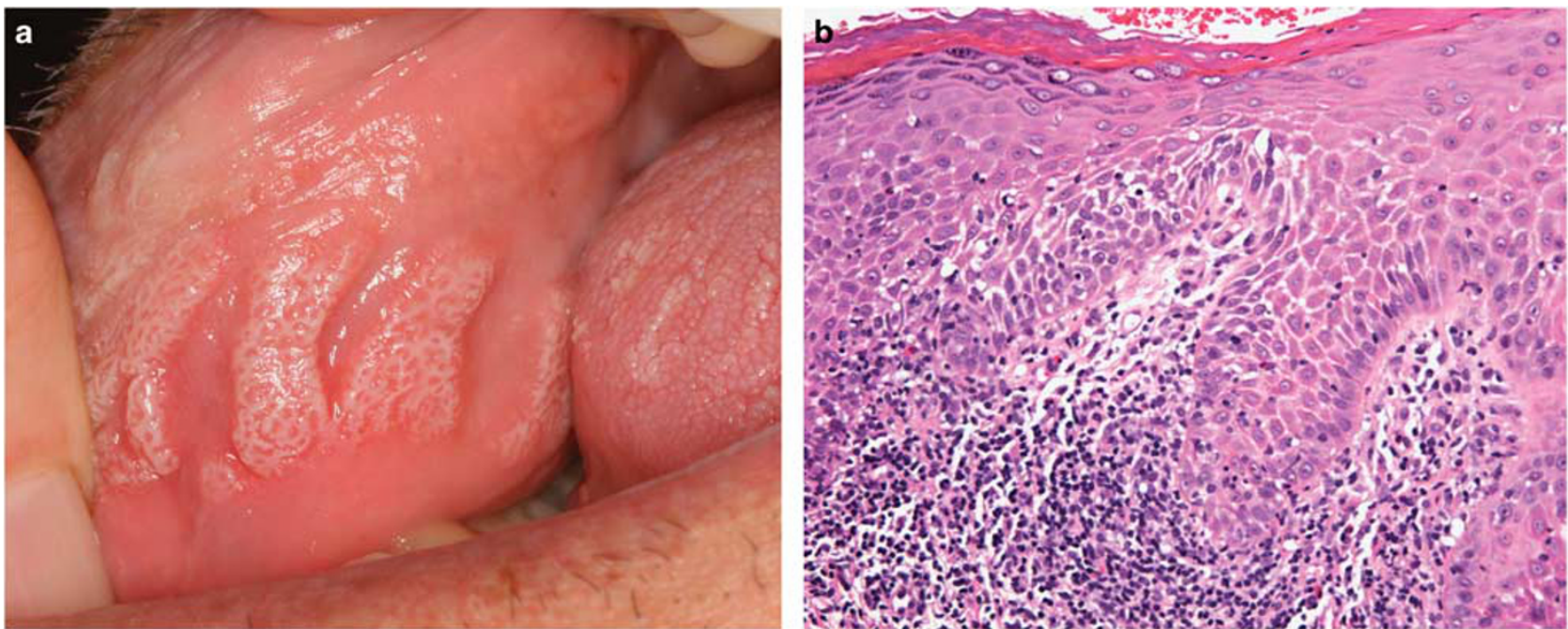

Figure 7 Oral lichenoid contact reaction to cinnamon flavored chewing gum. Within 10 days of discontinuing the gum, the lesion completely resolved. (a) The microscopic features of oral lichenoid contact reaction to cinnamon show marked epithelial acanthosis with elongation of the rete. Perivascular inflammatory cell infiltrate can be present as well as an occasional eosinophil (b, H\&E stain, original magnification $\times 250$ ).

are present in practically all cases of systemic lupus erythematosus, while direct immunofluorescence is positive in $\sim 70 \%$ of tissue samples from discoid lupus erythematosus. Indirect immunofluorescence is generally negative in discoid lupus erythematosus while antinuclear antibodies (ANA) are a serological hallmark in systemic lupus erythematosus.

Chronic graft versus host disease is a serious complication following allogeneic hematopoietic stem cell transplantation (bone marrow transplantation). Up to $80 \%$ of graft recipients may develop chronic graft versus host disease, usually within the first 6-24 months following transplantation. ${ }^{1,44}$ The most commonly affected areas are the skin, liver, oral cavity, and eyes, and in some cases oral chronic graft versus host disease may be the only affected anatomic site. ${ }^{45}$ Oral chronic graft versus host disease can have a variety of presentations, some of which overlap clinically with oral lichen planus. The reticular form is most common, with or without erosions mimicking oral lichen planus. Patients diagnosed with chronic graft versus host disease have an increased risk for the development of oral cancer and should be screened yearly. ${ }^{46,47}$ 
Appropriate clinical history is necessary in formulating a diagnosis as oral lichen planus and chronic graft versus host disease have overlapping histology. ${ }^{48,49}$ Numerous colloid bodies can be present along with basal cell degeneration. The inflammatory infiltrate in chronic graft versus host disease may be mixed containing plasma cells and eosinophils and also may not be as intense as in oral lichen planus. Direct immunofluorescence findings are similar to oral lichen planus and indirect immunofluorescence is negative.

Chronic ulcerative stomatitis is a rare mucocutaneous disease that can mimic both erosive oral lichen planus and mucous membrane pemphigoid. First described in 1990, the exact incidence is unknown with around 50 reported cases to date. ${ }^{50-52}$ Similar to oral lichen planus, chronic ulcerative stomatitis affects mainly women in the fifth to sixth decade. Chronic ulcerative stomatitis mostly involves the gingiva, tongue, and buccal mucosa but can affect all oral anatomic sites. Gingival involvement presents as desquamative gingivitis indistinguishable from oral lichen planus and mucous membrane pemphigoid. ${ }^{50,52}$

Histologically, no unique features are present to allow for differentiation of chronic ulcerative stomatitis from oral lichen planus. A band-like predominately lymphocytic infiltrate, hydropic basal cell degeneration, cytoid bodies, and atrophic stratified squamous epithelium is seen. ${ }^{52,53}$ Fortunately, direct immunofluorescence can separate chronic ulcerative stomatitis from oral lichen planus. Direct immunofluorescence of perilesional tissue in chronic ulcerative stomatitis demonstrates IgG antibodies in the nuclei of basal and parabasal epithelial cell in a speckled and/or granular pattern known as the stratified epithelium specific-ANA pattern. ${ }^{50,51,53}$ Some autoimmune diseases including lupus erythematosus, scleroderma, CREST syndrome (calcinosis, Raynaud's phenomenon, esophageal involvement, sclerodactyly, and telangiectasia) have an ANA pattern in epithelia; however, unlike chronic ulcerative stomatitis, the autoantibody deposits are present in the spinous layer. Nevertheless, tangential cutting may create difficulties in interpretation. A shaggy fibrin band at the BMZ can also be seen on direct immunofluorescence similar to oral lichen planus. ANA-SES is identified by indirect immunofluorescence using either guinea pig or monkey esophagus as a tissue substrate in chronic ulcerative stomatitis. ${ }^{50}$

\section{Oral lichen planus and potentially malignant lesions}

\section{Oral Epithelial Dysplasia with Lichenoid Features}

The malignant potential of oral lichen planus has been debated since the first report in 1924 and to date the controversy is unresolved. ${ }^{6}$ A recently published meta-analysis and systemic review of malignant transformation rates of oral lichen planus evaluating 16 studies with a total of 7806 patients had an overall average rate of $1.09 \% .^{7}$ Of the 88 patients who developed oral squamous cell carcinoma, the most common oral anatomic site was the tongue $(51 \%)$ followed by the buccal mucosa (32\%). The female to male ratio of 3:1 was similar in both the oral lichen planus group and the subset of patients who developed squamous cell carcinoma. This finding is opposite from conventional oral squamous cell carcinoma where the female to male ratio is 1:3. The average age of the oral lichen planus patients when they developed squamous cell carcinoma was almost 10 years older than the non-cancer
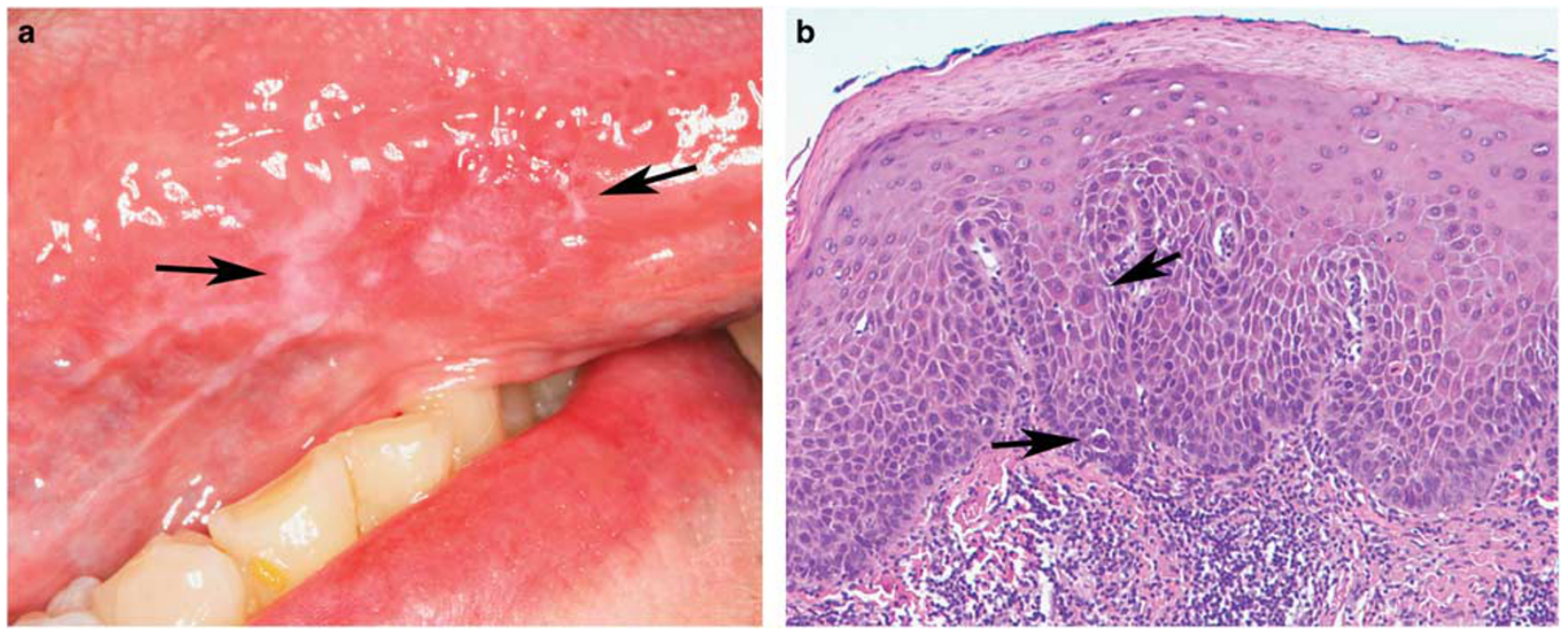

Figure 8 Clinical features of epithelial dysplasia mimicking oral lichen planus. A red and white area (arrows) was seen in the right lateral tongue of a 45-year-old female. The central atrophic area surrounded by a keratotic area was interpreted by the surgeon as oral lichen planus (a). The biopsy demonstrated high-grade dysplasia with numerous mitoses (arrows) (b, H\&E stain, original magnification $\times 250$ ). 
oral lichen planus group (60 years of age versus 51 years of age). ${ }^{7}$

To date, it is unclear whether oral lichen planus is an independent risk factor for malignant transformation. Separating confounding risk factors for oral squamous cell carcinoma including tobacco use when evaluating studies is unrealistic as many studies have not recorded secondary risk factors. Numerous studies have investigated various mechanisms involved in carcinogenesis including p53, PCNA, loss of heterozygosity at the tumor suppressor gene loci, and cytogenetic abnormalities. ${ }^{54-66}$ None of the data show convincing or consistent findings of the premalignant potential of oral lichen planus. The inflammatory cell infiltrate associated with oral lichen planus has been proposed to be a mechanism for malignant transformation. ${ }^{67}$ This proposal is not without merit as other chronic inflammatory diseases have been linked to cancer such as colon cancer in long-standing inflammatory bowel disease precipitated by intestinal microflora. ${ }^{68}$ Conversely, other data suggest that inflammatory and immune systems may inhibit tumorigenesis.

Clinically, oral leukoplakia may present with lichenoid features and a clinician may biopsy the area to confirm the clinical impression of oral lichen planus (Figure 8a and b). Oftentimes, biopsies of oral epithelial dysplasia can mimic oral lichen planus on low-power microscopy exhibiting a prominent bandlike chronic inflammatory infiltrate subjacent to the basal cells (Figure 9a and b). ${ }^{1}$ The pathologist is then confronted with a clinical impression of oral lichen planus and a low-power histology of oral lichen planus, and may be lulled into making the diagnosis of oral lichen planus without further investigation. However, it is important to remember that oral lichen planus tends to present as symmetrical multifocal lesions as opposed to the typically isolated lesion of oral epithelial dysplasia., ${ }^{1,2} \mathrm{~A}$ recent study examined the incidence of lichenoid features in oral epithelial dysplasia and oral squamous cell carcinoma including 'saw-tooth' rete ridges, interface mucositis, colloid bodies, and basal cell degeneration. ${ }^{12}$ Lichenoid features were present in $29 \%$ of the 352 oral epithelial dysplasia or squamous cell carcinoma and the most frequently encountered features were a band-like inflammatory

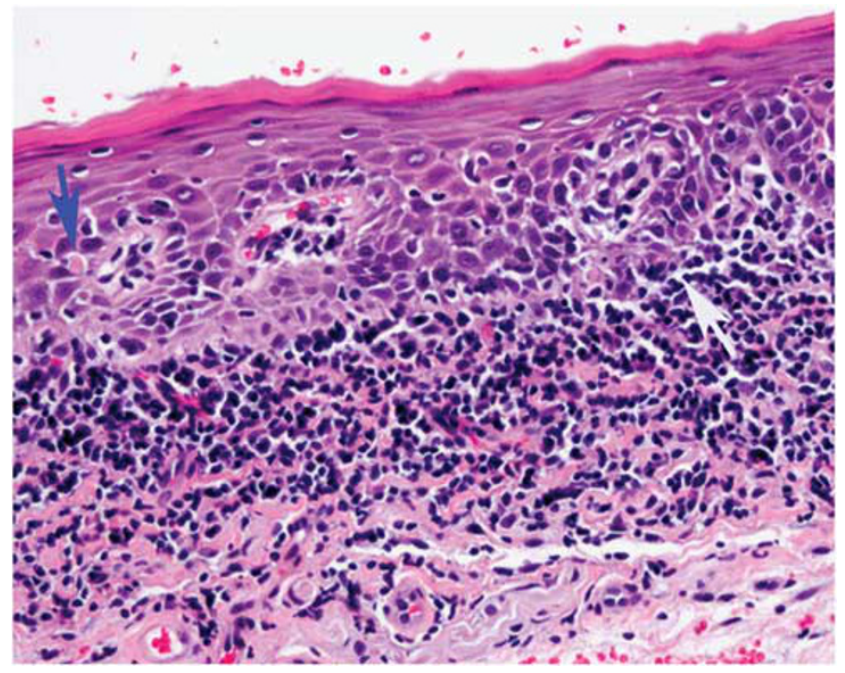

Figure 10 Epithelial dysplasia histologically mimicking atrophic oral lichen planus. A band-like predominately lymphocytic infiltrate as well as basal cell degeneration (white arrow) and colloid bodies (blue arrow) are seen in this biopsy from the lateral tongue. An increased nuclear-to-cytoplasmic ratio is present along with abnormal epithelial maturation. This should be diagnosed as moderate dysplasia and use of the term lichenoid dysplasia is to be discouraged. (H\&E stain, original magnification $\times 400$ ).
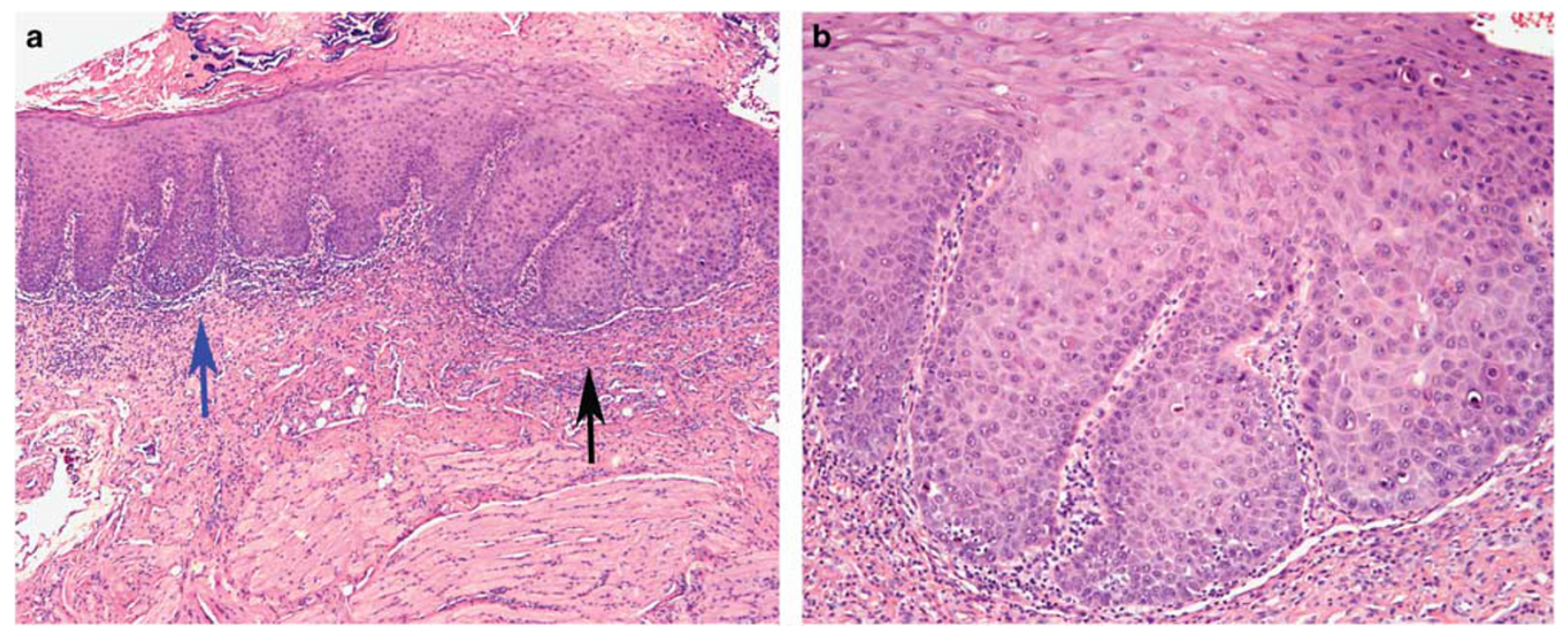

Figure 9 Histologic features of a case of epithelial dysplasia with lichenoid features. On low power magnification the biopsy of this case showed a 'lichenoid' appearance with a band-like inflammatory cell infiltrate (blue arrow) (a, H\&E stain, original magnification $\times 100)$. On higher magnification of the highlighted black arrow in a, hyperchromatic nuclei and significant cellular atypia are evident, but basal cell degeneration is not present $(\mathbf{b}, \mathrm{H} \& \mathrm{E}$ stain, original magnification $\times 250)$. 

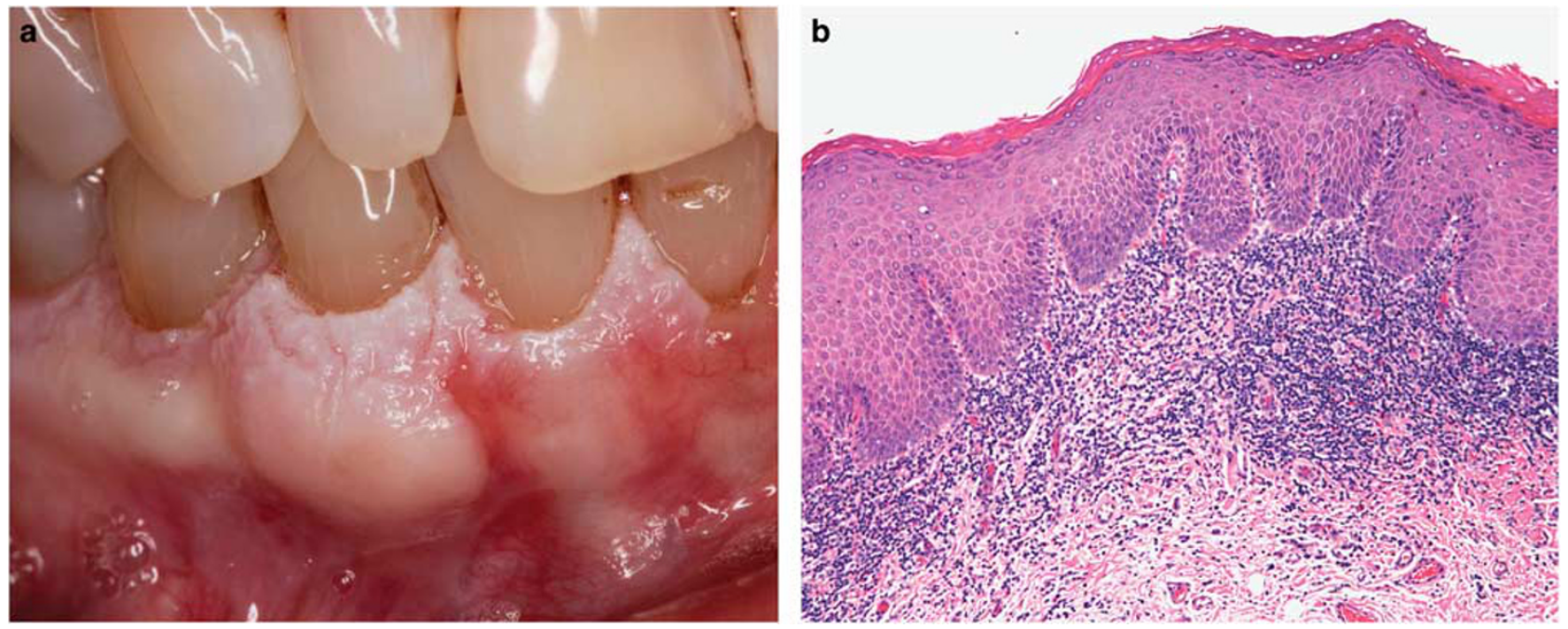

Figure 11 Clinical and histopathological features of proliferative verrucous leukoplakia in a 74-year-old non-smoking female. White, thickened plaques with irregular, rough surface change are noted on the gingiva of the mandible. The patient had other sites of involvement as well. (a) Biopsy showed hyperorthokeratosis, a prominent granular cell layer, a verrucoid epithelial architecture associated with interface mucositis. Absence of basal cell degeneration is noted (b, H\&E stain, original magnification $\times 100$ ).
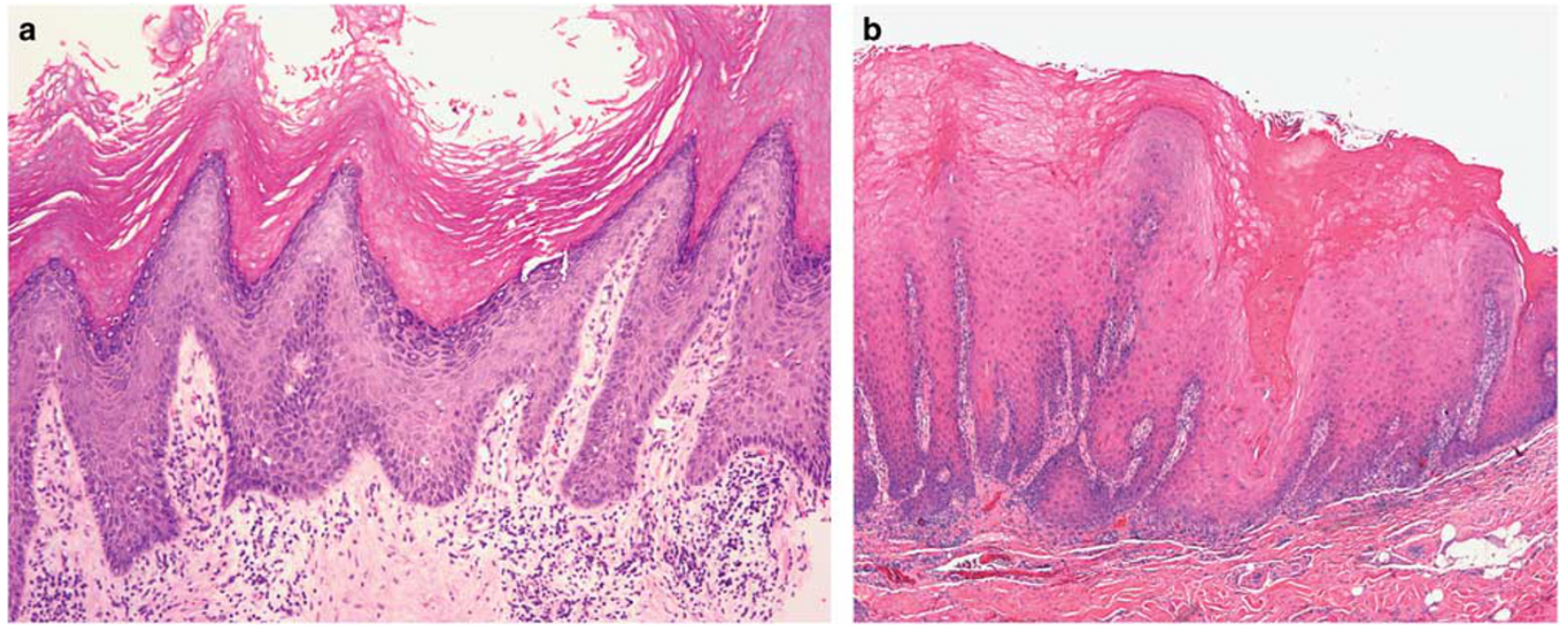

Figure 12 Proliferative verrucous leukoplakia can present with varied histopathology corresponding to the clinical presentation. In early stage disease verruciform epithelial orthokeratosis without dysplasia with or without focal interface mucositis can be encountered. These benign features underestimate the risk of malignant transformation in patients with proliferative verrucous leukoplakia (a, H\&E stain, original magnification $\times 100$ ). As the disease progresses the epithelium can become markedly hyperkeratotic with a verrucous architecture termed atypical verrucous hyperplasia. Proliferative verrucous leukoplakia demonstrates a relentless progression to either verrucous squamous cell carcinoma or squamous cell carcinoma (b, H\&E stain, original magnification $\times 100$ ).

cell infiltrate $(74 \%)$ and basal cell degeneration $(30 \%){ }^{12}$ On closer microscopic examination, the classic features of oral epithelial dysplasia including irregular epithelial stratification, loss of basal cell polarity, drop-shaped rete ridges, increased number of mitotic figures, premature keratinization, and hyperchromasia can be detected (Figure 10). This author acknowledges that the presence of intense inflammation can result in cytologic reactive atypia in oral lichen planus and distinguishing oral lichen planus and mild dysplasia can be subjective.
However, objective criteria such as a lymphocyte predominate infiltrate versus mixed inflammatory infiltrate can be at times helpful. ${ }^{1,2,13}$ Direct immunofluorescence cannot be used to distinguish oral epithelial dysplasia from oral lichen planus. Direct immunofluorescence findings of fibrinogen and/or C3 deposition at the BMZ has been described in $43 \%$ of oral epithelial dysplasia or oral squamous cell carcinoma similar to oral lichen planus. ${ }^{31}$ Grading oral epithelial dysplasia should be based on the degree of dysplasia (mild, moderate, and severe) 
rather than using the term lichenoid dysplasia. This term as a diagnosis should be discouraged, as it may create confusion resulting in suboptimal treatment.

An unusual and rare type of oral leukoplakia is proliferative verrucous leukoplakia, a distinct and aggressive form of oral precancer., ${ }^{1,2,14,15}$ Proliferative verrucous leukoplakia is associated with high recurrence and malignant transformation rates. Proliferative verrucous leukoplakia is mostly seen in older women ( $>60$ years of age; F:M ratio of $4: 1){ }^{14,15}$ The etiology of proliferative verrucous leukoplakia is unknown and is not associated with the usual risk factors for oral cancer including tobacco and alcohol use, and no association with HPV or other viruses have been detected. Proliferative verrucous leukoplakia, similar to oral lichen planus, is a multifocal disease affecting the gingiva, alveolar mucosa, buccal mucosa, palate, and dorsal tongue. ${ }^{14,15,69}$ The ventral tongue and floor of mouth are unusual sites of proliferative verrucous leukoplakia. The clinical presentation of multiple white keratotic plaques can be clinically mistaken for oral lichen planus and oftentimes proliferative verrucous leukoplakia is a diagnosis made in retrospect, particularly in its early stages. The clinical features of proliferative verrucous leukoplakia range from a focal flat white keratosis that with time becomes more diffuse (Figure 11a). Lesions may progress to a warty or verrucoid surface and have erythema, and ultimately can progress to either verrucous squamous cell carcinoma or squamous cell carcinoma. ${ }^{69}$ Histologically, proliferative verrucous leukoplakia biopsies, particularly in the early stages lack dysplasia but may have interface mucositis mimicking oral lichen planus (Figure 11b). However, there are some distinct histologic features of proliferative verrucous leukoplakia that aid in differentiating from oral lichen planus. Proliferative verrucous leukoplakia often exhibits verruciform epithelial hyperkeratosis, more often orthokeratosis rather than parakeratosis (Figure 12a and b). Progressive lesions may have dysplasia which precludes the diagnosis of oral lichen planus. Direct immunofluorescence cannot be used to differentiate proliferative verrucous leukoplakia from oral lichen planus. Atypical verrucous hyperplasia/hyperkeratosis shows fibrinogen deposition at the BMZ in $\sim 42 \%$ of cases and $3 \%$ of cases have both fibrinogen and C3 deposition. ${ }^{31}$

\section{Conclusion}

Owing to the tremendous overlap in the clinical and pathologic presentation of inflammatory, reactive, immune-mediated, and potentially premalignant lesions that affect the oral mucosa, oral lichenoid lesions can be a diagnostic challenge. Clinical information is essential as an accurate diagnosis cannot be made in a vacuum. Immunofluorescence may be a helpful adjunct in diagnosing some of the lichenoid lesions such as lupus and chronic ulcerative stomatitis. The presence of dysplasia should preclude the diagnosis of oral lichen planus or other benign lichenoid lesions to ensure appropriate patient management.

\section{Disclosure/conflict of interest}

The author declares no conflict of interest.

\section{References}

1 Muller S. Oral manifestations of dermatologic disease: a fochronic ulcerative stomatitis on lichenoid lesions. Head Neck Pathol 2011;5:36-40.

2 Muller S. The lichenoid tissue reactions of the oral mucosa: oral lichen planus and other lichenoid lesions. Surg Pathol Clinics 2011;4:1005-1026.

3 Khudhur AS, Di Zenzo G, Carrozzo M. Oral lichenoid tissue reactions: diagnosis and classification. Expert Rev Mol Diagn 2014;14:169-184.

4 Schlosser BJ. Lichen planus and lichenoid reactions of the oral mucosa. Dermatol Ther 2010;23:251-267.

5 Lodi G, Scully C, Carrozzo M et al. Current controversies in oral lichen planus: report of an international consensus meeting. Part 2. Clinical management and malignant transformation. Oral Surg Oral Med Oral Pathol Oral Radiol Endod 2005;100:164-178.

6 Montgomery D, Caulver G. Lichen planus of the mouth alone. Br J Dermatol 1924;41:45-50.

7 Fitzpatrick SG, Hirsch SA, Gordon SC. The malignant transformation of oral lichen planus and oral lichenoid lesions: a systematic review. J Am Dent Assoc 2014; 145:45-56.

8 Gonzalez-Moles MA, Scully C, Gil-Montoya JA. Oral lichen planus: controversies surrounding malignant transformation. Oral Dis 2008;14:229-243.

9 Larsson A, Warfvinge G. Malignant transformation of oral lichen planus. Oral Oncol 2003;39:630-631.

10 Mattsson U, Jontell M, Holmstrup P. Oral lichen planus and malignant transformation: is a recall of patients justified? Crit Rev Oral Biol Med 2002;13:390-396.

11 van der Meij EH, Schepman KP, Smeele LE et al. A review of the recent literature regarding malignant transformation of oral lichen planus. Oral Surg Oral Med Oral Pathol Oral Radiol Endod 1999;88:307-310.

12 Fitzpatrick SG, Honda KS, Sattar A et al. Histologic lichenoid features in oral dysplasia and squamous cell carcinoma. Oral Surg Oral Med Oral Pathol Oral Radiol 2014;117:511-520.

13 Krutchkoff DJ, Eisenberg E. Lichenoid dysplasia: a distinct histopathologic entity. Oral Surg Oral Med Oral Pathol 1985;60:308-315.

14 Gillenwater AM, Vigneswaran N, Fatani H et al. Proliferative verrucous leukoplakia: recognition and differentiation from conventional leukoplakia and mimics. Head Neck 2014;36:1662-1668.

15 Gillenwater AM, Vigneswaran N, Fatani $\mathrm{H}$ et al. Proliferative verrucous leukoplakia (PVL): a review of an elusive pathologic entity!. Adv Anat Pathol 2013;20: 416-422.

16 Gorouhi F, Davari P, Fazel N. Cutaneous and mucosal lichen planus: a comprehensive review of clinical 
subtypes, risk factors, diagnosis, and prognosis. Sci World J 2014;2014:742826.

17 Schifter M, Fernando SL, Li J. Oral Lichen Planus, Skin Biopsy - Diagnosis and Treatment. In: Suran F (ed.), InTech 2013; doi: 10.5772/56482; available from: http:// www.intechopen.com/books/skin-biopsy-diagnosis-andtreatment/oral-lichen-planus.

18 Parashar P. Oral lichen planus. Otolaryngol Clin North Am 2011;44:89-107, vi.

$19 \mathrm{Au}$ J, Patel D, Campbell JH. Oral lichen planus. Oral Maxillofac Surg Clin North Am 2013;25:93-100, vii.

20 Scully C, de Almeida OP, Welbury R. Oral lichen planus in childhood. Br J Dermatol 1994;130:131-133.

21 Pandhi D, Singal A, Bhattacharya SN. Lichen planus in childhood: a series of 316 patients. Pediatr Dermatol 2014;31:59-67.

22 De Rossi SS, Ciarrocca K. Oral lichen planus and lichenoid mucositis. Dent Clin North Am 2014;58: 299-313.

23 Crincoli V, Di Bisceglie MB, Scivetti M et al. Oral lichen planus: update on etiopathogenesis, diagnosis and treatment. Immunopharmacol Immunotoxicol 2011;33:11-20.

24 Andreasen JO. Oral lichen planus. 1. A clinical evaluation of 115 cases. Oral Surg Oral Med Oral Pathol 1968;25:31-42.

25 Thorn JJ, Holmstrup P, Rindum J et al. Course of various clinical forms of oral lichen planus. A prospective follow-up study of 611 patients. J Oral Pathol 1988;17:213-218.

26 Cheng YSL, Gould A, Kurago Z et al. Diagnosis of oral lichen planus: a position paper from the American Academy of Oral and Maxillofacial Pathology. Oral Surg Oral Med Oral Pathol Oral Radiol; advance online publication, 4 May 2016; doi:10.1016/j.oooo.2016.05. 004 (e-pub ahead of print).

27 van der Meij EH, van der Waal I. Lack of clinicopathologic correlation in the diagnosis of oral lichen planus based on the presently available diagnostic criteria and suggestions for modirect immunofluorescenceications. J Oral Pathol Med 2003;32:507-512.

28 Patterson J. The lichenoid reaction pattern ('interface dermatitis'). Weedon's Skin Pathology, 4th edn. Churchill Livingston Elsevier: London, UK, 2016, p 39.

29 Suresh L, Neiders ME. Definitive and differential diagnosis of desquamative gingivitis through direct immunofluorescence studies. J Periodontol 2012;83:1270-1278.

30 Morrison LH. Direct immunofluorescence microscopy in the diagnosis of autoimmune bullous dermatoses. Clin Dermatol 2001;19:607-613.

31 Montague LJ, Bhattacharyya I, Islam MN et al. Direct immunofluorescence testing results in cases of premalignant and malignant oral lesions. Oral Surg Oral Med Oral Pathol Oral Radiol 2015;119:675-683.

32 Scully C, Lo Muzio L. Oral mucosal diseases: mucous membrane pemphigoid. Br J Oral Maxillofac Surg 2008;46:358-366.

33 Zillikens D. Acquired skin disease of hemidesmosomes. J Dermatol Sci 1999;20:134-154.

34 Schmidt E, Zillikens D. Pemphigoid diseases. Lancet 2013;381:320-332.

35 Hayakawa T, Furumura M, Fukano H et al. Diagnosis of oral mucous membrane pemphigoid by means of combined serologic testing. Oral Surg Oral Med Oral Pathol Oral Radiol 2014;117:483-496.

36 Yuan A, Woo SB. Adverse drug events in the oral cavity. Oral Surg Oral Med Oral Pathol Oral Radiol 2015;119:35-47.
37 Issa Y, Duxbury AJ, Macfarlane TV et al. Oral lichenoid lesions related to dental restorative materials. Br Dent J 2005;198:361-366 disussion 549; quiz 372.

38 Thornhill MH, Sankar V, Xu XJ et al. The role of histopathological characteristics in distinguishing amalgam-associated oral lichenoid reactions and oral lichen planus. J Oral Pathol Med 2006;35:233-240.

39 Allen CM, Blozis GG. Oral mucosal reactions to cinnamon-flavored chewing gum. J Am Dent Assoc 1988;116:664-667.

40 Miller RL, Gould AR, Bernstein ML. Cinnamoninduced stomatitis venenata, Clinical and characteristic histopathologic features. Oral Surg Oral Med Oral Pathol 1992;73:708-716.

41 Walling HW, Sontheimer RD. Cutaneous lupus erythematosus: issues in diagnosis and treatment. Am J Clin Dermatol 2009;10:365-381.

42 Brennan MT, Valerin MA, Napenas JJ et al. Oral manifestations of patients with lupus erythematosus. Dent Clin North Am 2005;49:127-141, ix.

43 Farthing PM, Speight PM. Problems and pitfalls in oral mucosal pathology. Mini-symposium: head and neck pathology. Curr Diagn Pathol 2006;12:66-74.

44 Demarosi F, Lodi G, Carrassi A et al. Clinical and histopathological features of the oral mucosa in allogeneic haematopoietic stem cell transplantation patients. Exp Oncol 2007;29:304-308.

45 Imanguli MM, Alevizos I, Brown $\mathrm{R}$ et al. Oral graftversus-host disease. Oral Dis 2008;14:396-412.

46 Demarosi F, Lodi G, Carrassi A et al. Oral malignancies following HSCT: graft versus host disease and other risk factors. Oral Oncol. 2005;41:865-877.

47 Kruse AL, Grätz KW. Oral carcinoma after hematopoietic stem cell transplantation-a new classification based on a literature review over 30 years. Head Neck Oncol 2009;1:29.

48 Mays JW, Fassil H, Edwards DA et al. Oral chronic graft-versus-host disease: current pathogenesis, therapy, and research. Oral Dis 2013;19:327-346.

49 Shulman HM, Kleiner D, Lee SJ et al. Histopathologic diagnosis of chronic graft-versus-host disease: National Institutes of Health Consensus Development Project on Criteria for Clinical Trials in Chronic Graft-versus-Host Disease: II. Pathology Working Group Report. Biol Blood Marrow Transplant 2006;12: 31-47.

50 Solomon LW. Chronic ulcerative stomatitis. Oral Dis 2008;14:383-389.

51 Carlson MW, Garlick JA, Solomon LW. Chronic ulcerative stomatitis: evidence of autoimmune pathogenesis. Oral Surg Oral Med Oral Pathol Oral Radiol Endod 2011;111:742-748.

52 Islam MN, Cohen DM, Ojha J et al. Chronic ulcerative stomatitis: diagnostic and management challengesfour new cases and review of literature. Oral Surg Oral Med Oral Pathol Oral Radiol Endod 2007;104: 194-203.

53 Qari H, Villasante C, Richert J et al. The diagnostic challenges of separating chronic ulcerative stomatitis from oral lichen planus. Oral Surg Oral Med Oral Pathol Oral Radiol 2015;120:622-627.

54 Zhang L, Michelsen C, Cheng X et al. Molecular analysis of oral lichen planus. A premalignant lesion? Am J Pathol 1997;151:323-327.

55 Thongprasom K, Mutirangura A, Cheerat S. Telomerase activity in oral lichen planus. J Oral Pathol Med 1998;27:395-398. 
56 Fujita H, Nagata M, Hoshina $\mathrm{H}$ et al. Clinical significance and usefulness of quantification of telomerase activity in oral malignant and nonmalignant lesions. Int J Oral Maxillofac Surg 2004;33:693-699.

57 Mattila R, Alanen K, Syrjanen S. DNA content as a prognostic marker of oral lichen planus with a risk of cancer development. Anal Quant Cytol Histol 2004;26: 278-284.

58 Femiano F, Scully C. DNA cytometry of oral leukoplakia and oral lichen planus. Med Oral Patol Oral Cir Bucal 2005;10(Suppl 1):E9-E14.

59 Kim J, Yook JI, Lee EH et al. Evaluation of premalignant potential in oral lichen planus using interphase cytogenetics. J Oral Pathol Med 2001;30:65-72.

60 Oluwadara O, Giacomelli L, Christensen R et al. LCK, survivin and PI-3K in the molecular biomarker profiling of oral lichen planus and oral squamous cell carcinoma. Bioinformation 2009;4:249-257.

61 Sousa FA, Paradella TC, Carvalho YR. Rosa lupus erythematosus. Immunohistochemical expression of PCNA, p53, bax and bcl-2 in oral lichen planus and epithelial dysplasia. J Oral Sci 2009;51:117-121.

62 Safadi RA, Al Jaber SZ, Hammad HM et al. Oral lichen planus shows higher expressions of tumor suppressor gene products of p53 and p21 compared to oral mucositis. An immunohistochemical study. Arch Oral Biol 2010;55:454-461.
63 Accurso BT, Warner BM, Knobloch TJ et al. Allelic imbalance in oral lichen planus and assessment of its classification as a premalignant condition. Oral Surg Oral Med Oral Pathol Oral Radiol Endod 2011;112: 359-366.

64 Oliveira Alves M, Balducci I, Rodarte Carvalho Y et al. Evaluation of the expression of p53, MDM2, and SUMO-1 in oral lichen planus. Oral Dis 2013;19:775-780.

65 Zargaran M, Jamshidi S, Eshghyar N et al. Suitability/ unsuitability of cell proliferation as an indicator of malignant potential in oral lichen planus: an immunohistochemical study. Asian Pac J Cancer Prev 2013;14: 6979-6983.

66 Pigatti FM, Taveira LA, Soares CT. Immunohistochemical expression of Bcl-2 and Ki-67 in oral lichen planus and leukoplakia with different degrees of dysplasia. Int J Dermatol 2015;54:150-155.

67 Sonis ST, Amaral Mendes R. Could the PI3K canonical pathway be a common link between chronic inflammatory conditions and oral carcinogenesis? J Oral Pathol Med; advance online publication, 15 March 2016; doi:10.1111/jop.12436 (e-pub ahead of print).

68 Rakoff-Nahoum S. Why cancer and inflammation? Yale J Biol Med 2006;79:123-130.

69 van der Waal I, Reichart PA. Oral proliferative verrucous leukoplakia revisited. Oral Oncol 2008;4: $719-721$. 